\title{
Robust Nonlinear Position Control with Extended State Observer for Single-Rod Electro-Hydrostatic Actuator
}

\author{
Young Seop Son ${ }^{1}$ and Wonhee Kim ${ }^{2, *(D)}$ \\ 1 Department of Robot and Smart Systems Engineering, Kyungpook National University, \\ Daegu 702-701, Korea; ys.son@knu.ac.kr \\ 2 School of Energy Systems Engineering, Chung-Ang University, Seoul 156-756, Korea \\ * Correspondence: whkim79@cau.ac.kr; Tel.: +82-02-820-5928
}

Citation: Son, Y.S.; Kim, W. Robust Nonlinear Position Control with Extended State Observer for Single-Rod Electro-Hydrostatic Actuator. Mathematics 2021, 9, 2397. https://doi.org/10.3390/math9192397

Academic Editor: António M. Lopes

Received: 26 August 2021

Accepted: 22 September 2021

Published: 27 September 2021

Publisher's Note: MDPI stays neutral with regard to jurisdictional claims in published maps and institutional affiliations.

Copyright: (c) 2021 by the authors. Licensee MDPI, Basel, Switzerland. This article is an open access article distributed under the terms and conditions of the Creative Commons Attribution (CC BY) license (https:// creativecommons.org/licenses/by/ $4.0 /)$.

\begin{abstract}
In the existing literature, studies on position controller design using only position feedback, considering the disturbances for single-rod electro-hydrostatic actuators (EHAs), have not been reported. Herein, we propose a robust nonlinear position control with an extended state observer (ESO) for single-rod EHAs. A new EHA model that consists of position, velocity, and acceleration with an internal state variable is developed. Instead of the separated port pressure dynamics, the acceleration dynamics were defined. The external disturbance, model, and input function uncertainties were lumped into a disturbance. An ESO is developed to estimate the disturbance, as well as the position, velocity, and acceleration. In practice, it is difficult to accurately estimate the disturbance because it includes the external disturbance, system dynamics, and input function uncertainty. The poor estimation performance may degrade the position tracking performance, but a high gain cannot be used to suppress the estimation error because of the measurement noise amplification. To resolve this problem, a robust nonlinear position controller is developed via a backstepping procedure. In the controller, a nonlinear gain is implemented to sufficiently suppress position tracking without the use of a high gain. The stability of the closed-loop system is mathematically proven using the input-to-state stability. The proposed method is simple and suitable for real-time control.
\end{abstract}

Keywords: electro-hydrostatic actuator; position measurement; state estimation; position control

\section{Introduction}

In various industries, electro-hydraulic systems (EHSs) have been widely used as actuators because of their high power density, flexibility, and high stiffness, compared to their electrical counterparts [1]. Valve-controlled hydraulic systems are widely used, owing to their simplicity, but they have several problems such as low energetic efficiency (because of throttling losses), maintenance load, limited installation space, high weight, and environmental pollution (due to the leakage of the working fluid) [2]. To overcome these problems, electro-hydrostatic actuators (EHAs) have been developed by integrating an electric motor instead of a valve in valve-controlled hydraulic system. In EHAs, integrating the electric motor can lead to several advantages, such as smaller size, higher energy efficiency, and faster response due to the higher stiffness compared to valve-controlled hydraulic actuators. However, it is difficult to control the EHSs because the compressibility of the hydraulic fluid and the complex flow properties result in higher nonlinearities in their dynamics.

Various control methods have been proposed to improve the control performance of EHSs. A linear control method has been designed for hydraulic systems [1]. In [3,4], control methods using variable structure control (VSC) were developed for controlling the EHS. For disturbance compensation, a robust adaptive tracking control method was designed [5]. Control methods using input-output linearization have been developed to avoid the nonlinearities of EHSs [6-8]. A nonlinear control method was recently developed using the flatness property to improve the position-tracking performance [9]. The EHS 
model is in the form of strict feedback. Thus, various backstepping control methods have been developed using the EHS model properties [10-12]. An adaptive sliding mode control scheme was designed to resolve the nonlinearity problem of changes in the EHA [13]. In [14], a nonlinear adaptive position control method was developed based on a sliding mode back-stepping design method for the EHA. Although they improved the control performance, these methods require both full-state feedback and system parameter information. Thus, nonlinear controllers with observers have been designed to improve the control performance of the EHS using only output feedback $[15,16]$. However, external disturbances (i.e., load force and friction) and parameter uncertainties were not considered in previous methods. To resolve these problems, several control methods have been developed to address external disturbances and parameter uncertainties. In $[17,18]$, nonlinear control methods using singular perturbation theory were proposed for robustness against the effective bulk modulus uncertainty. Adaptation control methods have been established to compensate for uncertainties in EHS parameters [19-21]. Although these methods are robust against parameter uncertainties, they still require a full state. Furthermore, to the best of our knowledge, studies regarding position controller design using only position feedback, considering the disturbances for single-rod EHAs, have not been reported.

In this paper, we propose a robust nonlinear position control with an extended state observer (ESO) for single-rod EHAs. A new EHA model that consists of position, velocity, and acceleration with an internal state variable is proposed. The acceleration dynamics were defined instead of the separated port-pressure dynamics. The external disturbance, model, and input function uncertainties were lumped into a single disturbance. An ESO is developed to estimate the disturbance as well as the position, velocity, and acceleration. In practice, it is difficult to accurately estimate the disturbance because the disturbance includes the external disturbance, the system dynamics, and the input function uncertainty. Furthermore, poor estimation performance may degrade the position tracking performance, but the high gain to suppress the estimation error cannot be used because of the measurement noise amplification. To resolve this problem, a robust nonlinear position controller is developed via a backstepping procedure. In the controller, a nonlinear gain is implemented to sufficiently suppress position tracking without the use of a high gain. The stability of the closed-loop system is mathematically proven using the input-to-state stability (ISS). Only a nominal value of the input function is required in the proposed method. Thus, the proposed method is robust against the model uncertainties and the external disturbance. The proposed method is simple and suitable for real-time control. The performance of the proposed method is validated via simulations.

\section{System Model and Problem Formulation}

\subsection{Electro-Hydrostatic Actuator Model}

The structure of the single-rod electro-hydrostatic actuator is shown in Figure 1. The mathematical model of the EHA dynamics is represented as follows [16]:

$$
\begin{aligned}
& \dot{x}_{p}=x_{v} \\
& \dot{x}_{v}=\frac{1}{m}\left[-k x_{p}-b x_{v}+S_{A} P_{A}-S_{B} P_{B}-d_{L}+d_{F}\right] \\
& \dot{P}_{A}=\frac{\beta}{V_{A}\left(x_{p}\right)}\left[D_{p} \omega_{m}-S_{A} x_{v}-C_{p} P_{A}+C_{p} P_{B}\right] \\
& \dot{P}_{B}=\frac{\beta}{V_{B}\left(x_{p}\right)}\left[-D_{p} \omega_{m}+S_{B} x_{v}+C_{p} P_{A}-C_{p} P_{B}\right]
\end{aligned}
$$

where $x_{p}$ is the piston position $[\mathrm{m}], x_{v}$ is the piston velocity $[\mathrm{m} / \mathrm{s}], P_{A}$ and $P_{B}$ are the pressures of both chambers $\left[\mathrm{N} / \mathrm{m}^{2}\right], \omega_{m}$ is the rotational speed of the electric motor $[\mathrm{rad} / \mathrm{s}]$, $m$ is the total mass of the piston and load $[\mathrm{kg}], k$ is the load spring constant $[\mathrm{N} / \mathrm{m}], b$ is the viscous damping coefficient $[\mathrm{N} / \mathrm{m} / \mathrm{s}], d_{L}$ is the load force $[\mathrm{N}], d_{F}$ is the friction force $[\mathrm{N}]$, $D_{p}$ is the volumetric capacity of the pump $\left[\mathrm{m}^{3} / \mathrm{rad}\right], \beta_{e}$ is the effective bulk modulus of the 
system $\left[\mathrm{N} / \mathrm{m}^{2}\right], C_{p}$ is the leakage coefficient of the pump $\left[\mathrm{m}^{5} / \mathrm{Ns}\right]$, and $V_{A}\left(x_{p}\right)=V_{A 0}+$ $S_{A} x_{p}$ and $V_{B}\left(x_{p}\right)=V_{B 0}-S_{B} x_{p}$ are the volumes of chambers $\mathrm{A}$ and $\mathrm{B}\left[\mathrm{m}^{3}\right]$, respectively. In the EHA (1), $x_{p}$ is physically bounded as:

$$
-x_{p_{\lim }} \leq x_{p} \leq x_{p_{\lim }}
$$

where $x_{p_{\lim }}$ is a positive constant. The ranges of $V_{A}\left(x_{p}\right)$ and $V_{B}\left(x_{p}\right)$ are defined as:

$$
\begin{aligned}
V_{A 0}-S_{A} x_{p_{\lim }} & =V_{A_{\min }} \leq V_{A}\left(x_{p}\right) \leq V_{A_{\max }}=V_{A 0}+S_{A} x_{p_{\lim }} \\
V_{B 0}-S_{B} x_{p_{\text {lim }}} & =V_{B_{\min }} \leq V_{B}\left(x_{p}\right) \leq V_{B_{\max }}=V_{B 0}+S_{B} x_{p_{\lim }} .
\end{aligned}
$$

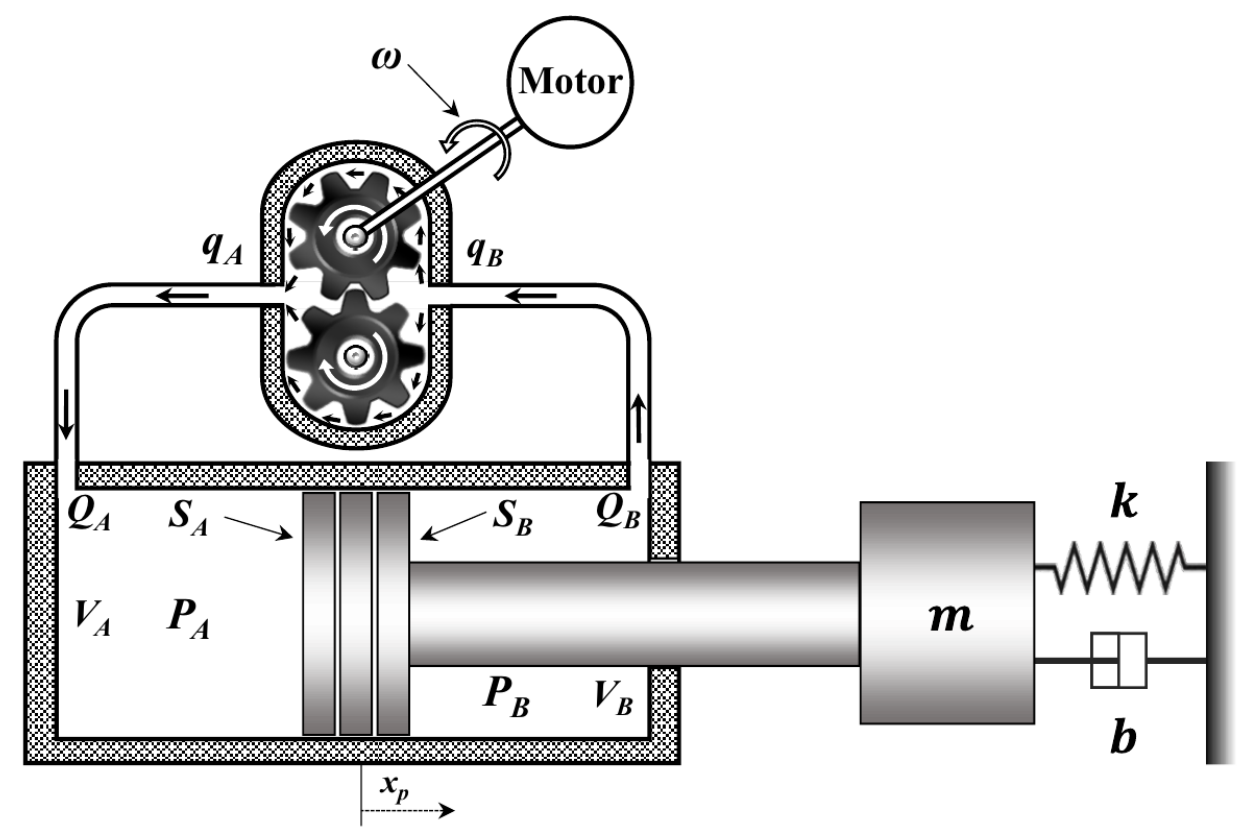

Figure 1. Structure of the single-rod electro-hydrostatic actuator.

For simplification, (1) can be rewritten as:

Assumption 1. The derivatives of the load force $F_{L}$ and friction $F_{F}$ with respect to time exist and are bounded.

\subsection{Problem Formulation}

In this paper, it is assumed that only the position is measurable. Now we define the new state variables $z$ and $x$ as:

$$
\begin{aligned}
& z=V_{A} P_{A}+V_{B} P_{B} \\
& x=\left[\begin{array}{l}
x_{1} \\
x_{2} \\
x_{3}
\end{array}\right]=\left[\begin{array}{l}
x_{p} \\
x_{v} \\
\dot{x}_{v}
\end{array}\right] .
\end{aligned}
$$

From (4) the relationships can be obtained as:

$$
\begin{aligned}
& x_{p}=x_{1} \\
& x_{v}=x_{2} \\
& P_{A}=\frac{V_{B} k x_{1}+V_{B} b x_{2}+V_{B} m x_{3}+S_{B} z+V_{B} d_{L}-V_{B} d_{F}}{S_{A} V_{B}+S_{B} V_{A}} \\
& P_{B}=\frac{-V_{A} k x_{1}-V_{A} b x_{2}-V_{A} m x_{3}+S_{A} z-V_{A} d_{L}+V_{B} d_{F}}{S_{A} V_{B}+S_{B} V_{A}} .
\end{aligned}
$$


From (1) and (5), we obtain the dynamics of $z$ and $x_{3}$ as:

$$
\begin{aligned}
\dot{z} & =\left(k x_{1}+b x_{2}+m x_{3}+d_{L}-d_{F}-\beta S_{A}+\beta S_{B}\right) x_{2} \\
\dot{x}_{3} & =\frac{1}{m}\left[-k x_{2}-b x_{3}+S_{A} \dot{P}_{A}-S_{B} \dot{P}_{B}-\dot{d}_{L}+\dot{d}_{F}\right] \\
& =f\left(z, x, \dot{d}_{L}, \dot{d}_{F}\right)+\underbrace{\beta D_{p}\left(\frac{S_{A}}{V_{A}\left(x_{1}\right)}+\frac{S_{B}}{V_{B}\left(x_{1}\right)}\right)}_{g\left(x_{1}\right)} u
\end{aligned}
$$

where $u$ is the control input and is defined as $u=\omega_{m}$. With (4) and (5), the EHA dynamics (1) are transformed into:

$$
\begin{aligned}
\dot{z} & =\left(k x_{1}+b x_{2}+m x_{3}+d_{L}-d_{F}-\beta S_{A}+\beta S_{B}\right) x_{2} \\
\dot{x}_{1} & =x_{2} \\
\dot{x}_{2} & =x_{3} \\
\dot{x}_{3} & =f\left(z, x, \dot{d}_{L}, \dot{d}_{F}\right)+g\left(x_{1}\right) u .
\end{aligned}
$$

Remark 1. In (7), $z$ is the internal state variable for the control of $x_{1}$. Generally, repetitive signals, i.e., sinusoidal signals or constant values are used for the desired position. Thus, the zero dynamics of $z$ are bounded. Consequently, only controls of $x_{1}, x_{2}$, and $x_{3}$ are required for position tracking control. In (7), $x_{1}, x_{2}$, and $x_{3}$ are controlled by the proposed control method.

Remark 2. In $g\left(x_{1}\right), D_{p}, S_{A}, S_{B}, V_{A}\left(x_{1}\right)$, and $V_{B}\left(x_{1}\right)$ can be easily known because they are mechanical fixed parameters. The effective bulk modulus $\beta$ is also a known and fixed parameter. However, in practice, $\beta$ is difficult to be exactly known. Furthermore, due to temperature variations and air entrapment, $\beta$ may vary during the operation of the hydraulic system [17].

The uncertainty and nominal value of $g\left(x_{1}\right)$ are defined as $\Delta g\left(x_{1}\right)$ and $g_{0}$ so that $g\left(x_{1}\right)=g_{0}+\Delta g\left(x_{1}\right)$. Because $V_{A}\left(x_{1}\right)$, and $V_{B}\left(x_{1}\right)$ are bounded, $\Delta g\left(x_{1}\right)$ is bounded.

Assumption 2. Among all parameters, only the nominal value of $g\left(x_{1}\right)$ is known.

Let us define the lumped disturbance $d$ as $d=f\left(z, x, \dot{d}_{L}, \dot{d}_{F}\right)+\Delta g\left(x_{1}\right) u$. The dynamics of $x$ are obtained as:

$$
\begin{aligned}
& \dot{x}_{1}=x_{2} \\
& \dot{x}_{2}=x_{3} \\
& \dot{x}_{3}=g_{0} u+d .
\end{aligned}
$$

The main goal in the controller design is the position tracking control using only $x_{1}$ feedback and $g_{0}$ information.

\section{Controller Design}

\subsection{Nonlinear Damping Position Controller}

For the controller design, we use the following assumption as follows:

Assumption 3. $x_{1}, x_{2}$, and $x_{3}$ are available. The estimated disturbance, $\hat{d}$ is bounded. 
$x_{1}, x_{2}, x_{3}$, and $d$ are estimated by the observer that will be designed in the next subsection. The tracking error $e=\left[\begin{array}{lll}e_{1} & e_{2} & e_{3}\end{array}\right]^{T}$ is defined as:

$$
\begin{aligned}
& e_{1}=x_{1}-x_{1_{d}} \\
& e_{2}=x_{2}-x_{2_{d}} \\
& e_{3}=x_{3}-x_{3_{d}}
\end{aligned}
$$

where $x_{1_{d}}=x_{p_{d}}$ is the desired position, and both the desired velocity, $x_{2_{d}}$ and desired acceleration, $x_{3_{d}}$ are yet to be defined. The tracking error dynamics are:

$$
\begin{aligned}
& \dot{e}_{1}=e_{2}+x_{2_{d}}-\dot{x}_{1_{d}} \\
& \dot{e}_{2}=e_{3}+x_{3_{d}}-\dot{x}_{2_{d}} \\
& \dot{e}_{3}=g_{0} u+d-\dot{x}_{3_{d}} .
\end{aligned}
$$

For the position tracking, the controller is designed as:

$$
\begin{aligned}
x_{2_{d}} & =-k_{1} e_{1}+\dot{x}_{1_{d}} \\
x_{3_{d}} & =-k_{2} e_{2}+\dot{x}_{2_{d}} \\
u & =\underbrace{\frac{1}{g_{0}}\left(-k_{3} e_{3}+\dot{x}_{3_{d}}-\hat{d}\right)}_{u_{a}}+\underbrace{\frac{1}{g_{0}}\left(k_{d}\left(\hat{e}_{1}, \hat{d}\right) e_{3}\right)}_{u_{b}} .
\end{aligned}
$$

where control gains, $k_{1}, k_{2}, k_{3}, k_{d_{1}}, k_{d_{2}}, v_{1}$ and $v_{2}$ are positive, $\hat{e}_{1}=\hat{x}_{1}-x_{1_{d}}$ is the estimated output tracking error, $\hat{x}_{1}$ is the estimation of $x_{1}$, and $k_{d}\left(\hat{e}_{1}, \hat{d}\right)=\left(k_{d_{1}} \sqrt{\hat{e}_{1}^{2}+v_{1}}+k_{d_{2}} \sqrt{\hat{d}^{2}+v_{2}}\right)$ is the nonlinear damping gain.

Remark 3. In (11), $u_{a}$ is used for stabilization and the other part $u_{b}$ is used to suppress the output tracking error as the estimation error of the disturbance is increased. In practice, because the external disturbances and system dynamics are lumped in the disturbance, it may be difficult to accurately estimate d. As long as the disturbance estimation error $\tilde{d}$ increases, the estimation error, estimated disturbance $\hat{d}$, and estimated output tracking error $\hat{x}_{1}$ may also increase. Thus, the nonlinear damping gain $k_{d}\left(\hat{e}_{1}, \hat{d}\right)$ is designed to suppress the tracking error when $\hat{e}_{1}$ and $\hat{d}$ are large.

Theorem 1. Suppose that the EHA dynamics (8). Under Assumptions 1-3, if the control law (11) is used to the EHA dynamics (8), then the tracking error has the following ISS properties:

$$
\begin{aligned}
& \left|e_{1}(t)\right| \leq \exp \left(-\frac{k_{1}}{2} t\right)\left|e_{1}(0)\right|+\frac{2}{k_{1}} \sup _{0 \leq \tau \leq t} e_{2}(\tau) \\
& \left|e_{2}(t)\right| \leq \exp \left(-\frac{k_{2}}{2} t\right)\left|e_{2}(0)\right|+\frac{2}{k_{2}} \sup _{0 \leq \tau \leq t} e_{3}(\tau) \\
& \left|e_{3}(t)\right| \leq \exp \left(-\frac{k_{3}}{2} t\right)\left|e_{3}(0)\right|+\frac{2}{k_{3}} \sup _{0 \leq \tau \leq t} \sigma(\tau) .
\end{aligned}
$$

where $\sigma=\frac{|d-\hat{d}|}{0.5 k_{3}+k_{d}\left(\hat{e}_{1}, \hat{d}\right)}$.

Proof. With control law (11), the tracking error dynamics (10) become:

$$
\begin{aligned}
& \dot{e}_{1}=-k_{1} e_{1}+e_{2} \\
& \dot{e}_{2}=-k_{2} e_{2}+e_{3} \\
& \dot{e}_{3}=-k_{3} e_{3}-k_{d}\left(\hat{e}_{1}, \hat{d}\right) e_{3}+d-\hat{d} .
\end{aligned}
$$


From (13), we have:

$$
\begin{aligned}
\frac{d}{d t}\left(\frac{e_{1}^{2}}{2}\right) & =-k_{1} e_{1}^{2}+e_{1} e_{2} \\
& \leq-\frac{k_{1}}{2} e_{1}^{2}-\frac{k_{1}}{2}\left|e_{1}\right|\left(\left|e_{1}\right|-\frac{2}{k_{1}}\left|e_{2}\right|\right) \\
\frac{d}{d t}\left(\frac{e_{2}^{2}}{2}\right) & =-k_{2} e_{2}^{2}+e_{2} e_{3} \\
& \leq-\frac{k_{2}}{2} e_{2}^{2}-\frac{k_{2}}{2}\left|e_{2}\right|\left(\left|e_{2}\right|-\frac{2}{k_{2}}\left|e_{3}\right|\right) .
\end{aligned}
$$

Then, the following results are derived as:

$$
\begin{aligned}
& \left|e_{1}(t)\right| \leq \exp \left(-\frac{k_{1}}{2} t\right)\left|e_{1}(0)\right|+\frac{2}{k_{1}} \sup _{0 \leq \tau \leq t} e_{2}(\tau) \\
& \left|e_{2}(t)\right| \leq \exp \left(-\frac{k_{2}}{2} t\right)\left|e_{2}(0)\right|+\frac{2}{k_{2}} \sup _{0 \leq \tau \leq t} e_{3}(\tau) .
\end{aligned}
$$

From (15) it is guaranteed that the relationships between $e_{1}$ and $e_{2}$, and $e_{2}$ and $e_{3}$ have ISS property. The dynamics of $e_{3}^{2}$ are:

$$
\begin{aligned}
\frac{d}{d t}\left(\frac{e_{3}^{2}}{2}\right) & =-k_{3} e_{3}^{2}-k_{d}\left(\hat{e}_{1}, \hat{d}\right) e_{3}^{2}+(d-\hat{d}) e_{3} \\
& \leq-\frac{k_{3}}{2} e_{3}^{2}-\left(\frac{k_{3}}{2}+k_{d}\left(\hat{e}_{1}, \hat{d}\right)\right)\left|e_{3}\right|\left(\left|e_{3}\right|-\sigma\right)
\end{aligned}
$$

Then,

$$
\left|e_{3}(t)\right| \leq \exp \left(-\frac{k_{3}}{2} t\right)\left|e_{3}(0)\right|+\frac{2}{k_{3}} \sup _{0 \leq \tau \leq t} \sigma(\tau) .
$$

(17) shows that the relationship between $e_{3}$ and $\sigma$ has the ISS property. From (15) and (17), the ISS property of the overall tracking error system is (12).

Remark 4. In (12), it is observed that $\sigma$ gets smaller simultaneously when $\hat{d}$ in the denominator of $\sigma$ becomes larger. In other words, the nonlinear damping in (11) increases so that the effects of $|d-\hat{d}|$ to $e_{3}$ can be sufficiently suppressed. Consequently, from the ISS property of the overall tracking error system (12), we can observe that the nonlinear damping gain $k_{d}\left(\hat{e}_{1}, \hat{d}\right)$ helps to suppress $\left|e_{1}\right|$.

\subsection{Extended State Observer Design}

In the previous subsection, the controller was designed using Assumption 3. In this subsection, the ESO is designed to estimate $x_{1}, x_{2}, x_{3}$, and $d$. Let us define $x_{4}$ as $x_{4}=d$. We define the extended state $x_{a}$ as:

$$
x_{a}=\left[\begin{array}{llll}
x_{1} & x_{2} & x_{3} & x_{4}
\end{array}\right]^{T} .
$$

The dynamics of $d$ can be defined as:

$$
\dot{d}=\delta .
$$


The estimated states, $\hat{x}$, and the estimated extended states, $\hat{x}_{a}$, are defined as:

$$
\begin{aligned}
\hat{x} & =\left[\begin{array}{lll}
\hat{x}_{1} & \hat{x}_{2} & \hat{x}_{3}
\end{array}\right]^{T} \\
\hat{x}_{a} & =\left[\begin{array}{llll}
\hat{x}_{1} & \hat{x}_{2} & \hat{x}_{3} & \hat{x}_{4}
\end{array}\right]^{T} .
\end{aligned}
$$

The ESO is developed as:

$$
\dot{\hat{x}}_{a}=A_{o} \hat{x}_{a}+L\left(x_{1}-\hat{x}_{1}\right)
$$

where $A_{o}=\left[\begin{array}{llll}0 & 1 & 0 & 0 \\ 0 & 0 & 1 & 0 \\ 0 & 0 & 0 & 1 \\ 0 & 0 & 0 & 0\end{array}\right]$ and $B_{0}=\left[\begin{array}{l}0 \\ 0 \\ 0 \\ 1\end{array}\right], L=\left[\begin{array}{llll}l_{1} & l_{2} & l_{3} & l_{4}\end{array}\right]^{T}$ is the observer gain matrix. The estimation errors of the states and the extended states, $\tilde{x}$ and $\tilde{x}_{a}$ are defined as:

$$
\begin{gathered}
\tilde{x}=\left[\begin{array}{l}
\tilde{x}_{1} \\
\tilde{x}_{2} \\
\tilde{x}_{3}
\end{array}\right]=\left[\begin{array}{l}
x_{1}-\hat{x}_{1} \\
x_{2}-\hat{x}_{2} \\
x_{3}-\hat{x}_{3}
\end{array}\right] \\
\tilde{x}_{a}=\left[\begin{array}{l}
\tilde{x}_{1} \\
\tilde{x}_{2} \\
\tilde{x}_{3} \\
\tilde{x}_{4}
\end{array}\right]=\left[\begin{array}{l}
x_{1}-\hat{x}_{1} \\
x_{2}-\hat{x}_{2} \\
x_{3}-\hat{x}_{3} \\
x_{4}-\hat{x}_{4}
\end{array}\right] .
\end{gathered}
$$

The dynamics of $\tilde{x}_{a}$ are:

$$
\dot{\tilde{x}}_{a}=\left(A_{o}-L C_{a}\right) \tilde{x}_{a}+B_{d} \delta
$$

where $B_{d}=\left[\begin{array}{llll}0 & 0 & 0 & 1\end{array}\right]^{T}$ and $C_{a}=\left[\begin{array}{llll}1 & 0 & 0 & 0\end{array}\right]$.

Assumption 4. The states are bounded, i.e., $\|x\|_{2} \leq B_{x}$ where $B_{x}$ is an unknown constant.

In most systems, all states are physically bounded [22]. Thus Assumption 4 is reasonable. The derivative of $\Delta g\left(x_{1}\right)$ is the function of $x_{2}$, thus, it is also bounded. Under Assumptions 1 and 4 , there exists an upper boundedness of $\delta,|\dot{d}|=\left|\dot{x}_{4}\right|=|\delta| \leq \delta_{\max }$.

Proposition 1. Let us consider the dynamics of $\tilde{x}_{a}$ (23). If the observer gains are chosen such that the roots of:

$$
s^{4}+l_{1} s^{3}+l_{2} s^{2}+l_{3} s+l_{4}=0 .
$$

are in the left-half plane, then $\tilde{x}_{a}$ is uniformly ultimately bounded.

Proof. We define the Lyapunov function $V_{o}$ as:

$$
V_{o}=\tilde{x}_{a}^{T} P_{o} \tilde{x}_{a}
$$

where $P_{o}$ is positive definite such that $\left(A_{a}-L C_{a}\right)^{T} P_{o}+P_{o}\left(A_{a}-L C_{a}\right)=-I$. The derivative of $V_{o}$ with respect to time is derived as:

$$
\begin{aligned}
\dot{V}_{o} & =\tilde{x}_{a}^{T}\left[\left(A_{a}-L C_{a}\right)^{T} P_{o}+P_{o}\left(A_{a}-L C_{a}\right)\right] \tilde{x}_{a}+2 \tilde{x}_{a}^{T} P_{o} B_{d} \delta \\
& \leq-\left\|\tilde{x}_{a}\right\|_{2}^{2}+2 \delta_{\max }\left\|P_{o}\right\|_{2}\left\|\tilde{x}_{a}\right\|_{2} \\
& \leq-\left\|\tilde{x}_{a}\right\|_{2}\left(\left\|\tilde{x}_{a}\right\|_{2}-2 \lambda_{\max }\left(P_{o}\right) \delta_{\max }\right)
\end{aligned}
$$

where $\lambda_{\max }\left(P_{o}\right)$ is the maximum eigenvalue of $P_{o}$. 
For $\left\|\tilde{x}_{a}\right\|_{2} \geq 2 \lambda_{\max }\left(P_{o}\right) \delta_{\max }$

$$
\dot{V}_{o} \leq-\left\|\tilde{x}_{a}\right\|_{2}^{2}
$$

Thus $\tilde{x}_{a}$ is uniformly ultimately bounded.

\subsection{Analysis of the Closed-Loop Stability}

In this subsection, the stability of the closed-loop system is examined. In (11), $\hat{x}$ is used instead of $x$. Thus, the actual control law is:

$$
\begin{aligned}
\hat{x}_{2_{d}} & =-k_{1} \hat{e}_{1}+\dot{x}_{1_{d}} \\
\hat{x}_{3_{d}} & =-k_{2} \hat{e}_{2}+\dot{x}_{2_{d}} \\
\hat{u} & =\frac{1}{g_{0}}\left(-k_{3} \hat{e}_{3}+\dot{\hat{x}}_{3_{d}}-\hat{d}-k_{d}\left(\hat{e}_{1}, \hat{d}\right) \hat{e}_{3}\right) .
\end{aligned}
$$

Note that $\hat{u}$ is used to avoid confusion between (11) and (28). Thus, $\hat{u}(28)$ is applied to the EHS, i.e., $\hat{u}=\omega$. With (28), the tracking error dynamics (10) become:

$$
\dot{e}=A_{e} e+B_{e} \xi
$$

where $A_{e}=\left[\begin{array}{ccc}-k_{1} & 0 & 0 \\ 0 & -k_{2} & 1 \\ 0 & 0 & -k_{3}\end{array}\right], B_{e}=\left[\begin{array}{l}0 \\ 0 \\ 1\end{array}\right]$, and $\xi(x, \hat{x})=k_{3} e_{3}+\hat{u}+d-\dot{\tau}_{a_{d}}$. From

and (29), the closed-loop system is:

$$
\begin{gathered}
\dot{e}=A_{e} e+B_{e} \xi \\
\dot{\tilde{x}}_{a}=A_{o} \tilde{x}_{a}+B_{d} \delta .
\end{gathered}
$$

Theorem 2. Let us consider the system (8). Under Assumptions 1, 2 and 4, if the output feedback controller (21) and (28) is implemented in the system (8), the following ISS properties for the tracking error hold:

$$
\begin{aligned}
& \left|e_{1}(t)\right| \leq \exp \left(-\frac{k_{1}}{2} t\right)\left|e_{1}(0)\right|+\frac{2}{k_{1}} \sup _{0 \leq \tau \leq t} e_{2}(\tau) \\
& \left|e_{2}(t)\right| \leq \exp \left(-\frac{k_{2}}{2} t\right)\left|e_{2}(0)\right|+\frac{2}{k_{2}} \sup _{0 \leq \tau \leq t} e_{3}(\tau) \\
& \left|e_{3}(t)\right| \leq \exp \left(-\frac{k_{3}}{2}(t)\right)\left|e_{3}(0)\right|+\sup _{0 \leq \tau \leq t} k_{\sigma_{1}}\left(k_{\tilde{x}}+1\right)\|\tilde{x}(\tau)\|
\end{aligned}
$$

where $k_{\sigma_{1}}=\frac{1}{0.5 k_{3}+k_{d}\left(\hat{e}_{1}, \hat{d}\right)}$.

Proof. In Proposition 1, it was proven that $\tilde{x}_{a}$ is uniformly ultimately bounded. In (12) of Theorem 1, it was shown that the tracking errors possess a cascade nature. Because the control input (28) that uses the estimation states, $\hat{x}$ is implemented in the $e_{3}$ subsystem of $e$, and it is sufficient to investigate the behavior of the $e_{3}$ subsystem owing to the cascade nature. In $u$ (11) and $\hat{u}$ (28), different desired velocities and actual torques are used respectively. However, the same desired position $x_{1_{d}}$ is used in both $u$ (11) and $\hat{u}$ (28). Thus, there exists $k_{\tilde{x}}>0$ such that:

$$
\left|\hat{u}\left(\hat{x}, x_{1_{d}}\right)-u\left(x, x_{1_{d}}\right)\right| \leq k_{\tilde{x}}\|\hat{x}-x\| .
$$

With $\hat{u}$, the dynamics of $e_{3}$ in (10) become:

$$
\dot{e}_{3}=-k_{3} e_{3}-k_{d}\left(\hat{e}_{1}, \hat{d}\right) e_{3}+d-\hat{d}+\hat{u}-u .
$$


Equation (33) can be obtained as:

$$
\frac{d}{d t}\left(\frac{e_{3}^{2}}{2}\right) \leq-\frac{k_{3}}{2} e_{3}^{2}+\left(\frac{k_{3}}{2}+k_{d}\left(\hat{e}_{1}, \hat{d}\right)\right)\left|e_{3}\right|\left(\left|e_{3}\right|-\sigma_{1}\right)
$$

where $\sigma_{1}=\frac{|d-\hat{d}|+\hat{u}-u}{0.5 k_{3}+k_{d}\left(\hat{e}_{1}, \hat{d}\right)}$. From (32),

$$
\begin{aligned}
\sigma_{1} & =\frac{|d-\hat{d}|+\hat{u}-u}{0.5 k_{3}+k_{d}\left(\hat{e}_{1}, \hat{d}\right)} \\
& \leq \frac{\|\tilde{x}\|+k_{\tilde{x}}\|\hat{x}-x\|}{0.5 k_{3}+k_{d}\left(\hat{e}_{1}, \hat{d}\right)} \\
& \leq k_{\sigma_{1}}\left(k_{\tilde{x}}+1\right)\|\tilde{x}\| .
\end{aligned}
$$

where $k_{\sigma_{1}}=\frac{1}{0.5 k_{3}+k_{d}\left(\hat{e}_{1}, \hat{d}\right)}$. Then,

$$
\left|e_{3}(t)\right| \leq \exp \left(-\frac{k_{3}}{2}(t)\right)\left|e_{3}(0)\right|+\frac{2}{k_{3}} \sup _{0 \leq \tau \leq t} k_{\sigma_{1}}\left(k_{\tilde{x}}+1\right)\|\tilde{x}(\tau)\| .
$$

Thus, the overall tracking error system has the ISS properties of a serial interconnected system (31).

Remark 5. As long as the disturbance increases, the estimation error $\tilde{x}$, estimated disturbance $\hat{d}$, and estimated output tracking error $e_{1}$ may also increase. It is observed that $k_{\sigma_{1}}$ decreases simultaneously when $k_{d}$ in the denominator of $k_{\sigma_{1}}$ increases. Thus, the tracking error is sufficiently suppressed. From the ISS properties of the serial interconnected system (31), we see that a high observer gain in the ESO is not necessary for a small output tracking error.

Figure 2 shows a block diagram of the proposed method. The ESO estimates $x_{1}, x_{2}, x_{3}$, and $d$ using only the output feedback $x_{p}$. The controller then generates the control input $\omega$ using the estimated states and the desired position $x_{p_{d}}$.

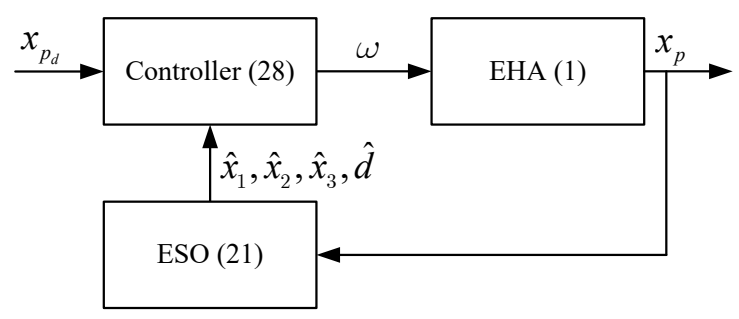

Figure 2. Block diagram of the proposed method.

\section{Simulations}

The performance of the proposed method was evaluated using simulations. In this paper, it was assumed that only $x_{1}$ and $g_{0}$ are known. Thus, only $x_{1}$ and $g_{0}$ were used for the proposed method in these simulations. The EHA and control parameters used are listed in Table 1. For comparison, two control methods were tested, i.e., the proposed method and the following PID controller:

$$
u=k_{P}\left(x_{1_{d}}-x_{1}\right)+k_{I} \int_{0}^{t}\left(x_{1_{d}}-x_{1}\right) d t+k_{D}\left(\dot{x}_{1_{d}}-\dot{x}_{1}\right)+k_{f} f \dot{x}_{1}
$$

where $k_{P}=5000, k_{I}=100, k_{D}=100$, and $k_{f f}=20$ are the control gains. These control gains were chosen with the consideration of the effects of $\mathrm{P}, \mathrm{I}$, and $\mathrm{D}$ tuning listed in Table 2 [23]. The control gains were tuned to obtain the best position tracking performances 
in both methods. In the simulations, the following load force and friction were used as external disturbances.

- $\quad$ Load force: $d_{L}=200$

- Friction: $d_{F}=\left(\alpha_{0}+\alpha_{1} \exp \left(-\frac{x_{v}}{v_{0}}\right)^{2}\right) \operatorname{sgn}\left(x_{v}\right)+\alpha_{2} x_{v}$.

Table 1. Parameters.

\begin{tabular}{cccc}
\hline Parameter & Value & Parameter & Value \\
\hline$k$ & 4000 & $b$ & 100 \\
$m$ & 80 & $S_{A}$ & $7.94226 \times 10^{-5}$ \\
$S_{B}$ & $5.43251 \times 10^{-5}$ & $D_{p}$ & $8.43521 \times 10^{-8}$ \\
$C_{p}$ & $2 \times 10^{-13}$ & $\beta_{e}$ & $1.8 \times 10^{9}$ \\
$k_{1}$ & 200 & $k_{2}$ & 400 \\
$k_{3}$ & 400 & $k_{d_{1}}$ & 100 \\
$k_{d_{2}}$ & 100 & $v_{1}$ & 1 \\
$v_{2}$ & 1 & $l_{1}$ & $5.0265 \times 10^{3}$ \\
$l_{2}$ & $9.4748 \times 10^{6}$ & $l_{3}$ & $7.9376 \times 10^{9}$ \\
$l_{4}$ & $2.4937 \times 10^{12}$ & & \\
\hline
\end{tabular}

Table 2. Effects of $P, I$, and D tuning.

\begin{tabular}{cccccc}
\hline Closed-Loop Response & Rise Time & Overshoot & Settling Time & Steady-State Error & Stability \\
\hline Increasing $K_{p}$ & Decrease & Increase & Small increase & Decrease & Degrade \\
\hline Increasing $K_{I}$ & Small decrease & Increase & Increase & Large decrease & Degrade \\
\hline Increasing $K_{D}$ & Small decrease & Decrease & Decrease & Minor change & Improve \\
\hline
\end{tabular}

The friction model used in the simulations includes the stiction, viscous damping, Coulomb friction, and Stribeck effect [24].

\subsection{Sinusoidal Desired Position Tracking}

The sinusoidal desired position $x_{1_{d}}=15 \sin (0.5 \pi t)$ depicted in Figure 3 was used. The estimation performances and errors of the ESO are shown in Figures 4 and 5 . The estimated states and disturbances satisfactorily follow the actual states and disturbances. The peaking phenomenon occurred because of the nonzero initial velocity at the desired position. However, the estimated states and disturbances converged rapidly to the actual states and disturbances. The position tracking errors of the PID controller and the proposed method are shown in Figure 6. The PID controller had a larger position-tracking error than the proposed method. At the outset, a large position tracking error appeared because of the nonzero initial velocity of the desired position. In the proposed method, the increased nonlinear damping gain suppressed the position tracking error. Thus, the proposed method was able to reduce the position-tracking error. Figure 7 shows the control input of the PID controller and the proposed method. At the outset, a slightly larger input was used in the proposed method compared to the PID controller because the nonlinear damping increased to suppress the position tracking error. After the position tracking error was sufficiently suppressed at $0.9 \mathrm{~s}$, the control input of the proposed method was similar to that of the PID controller. 


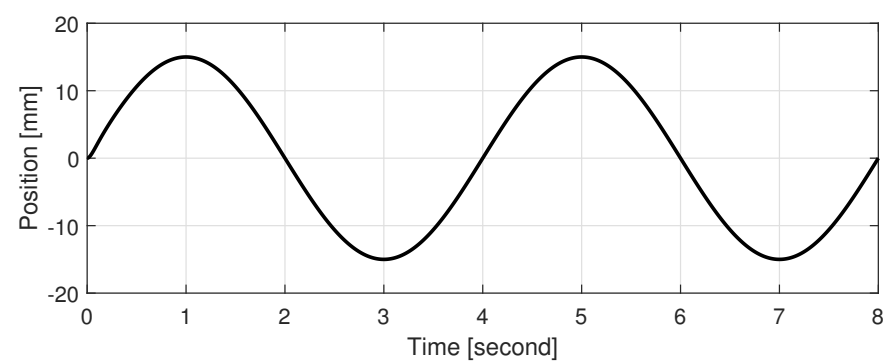

Figure 3. Desired position, $x_{1_{d}}$.

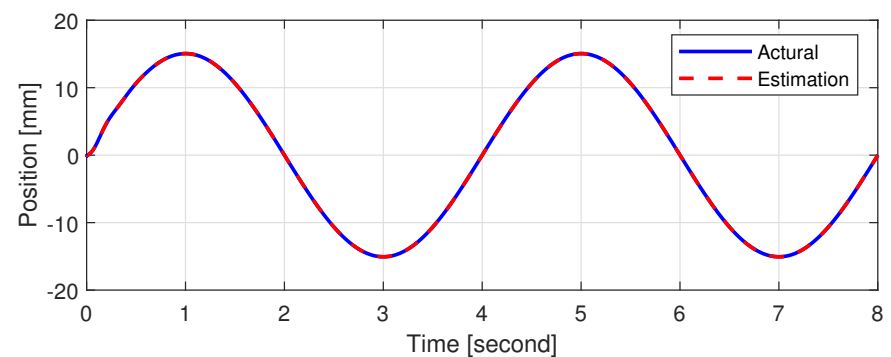

(a) Position, $x_{1}$

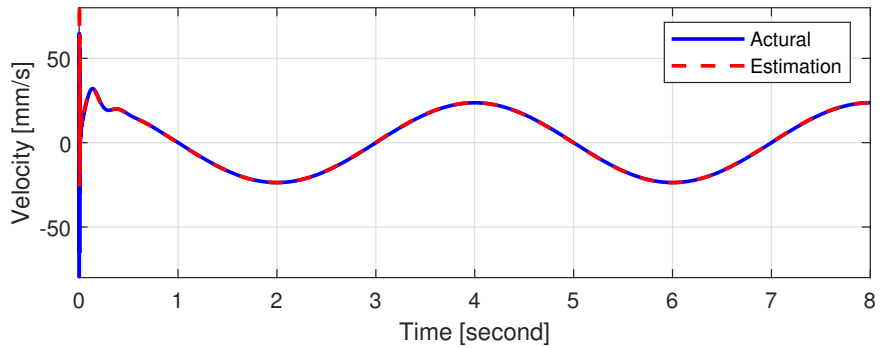

(b) Velocity, $x_{2}$

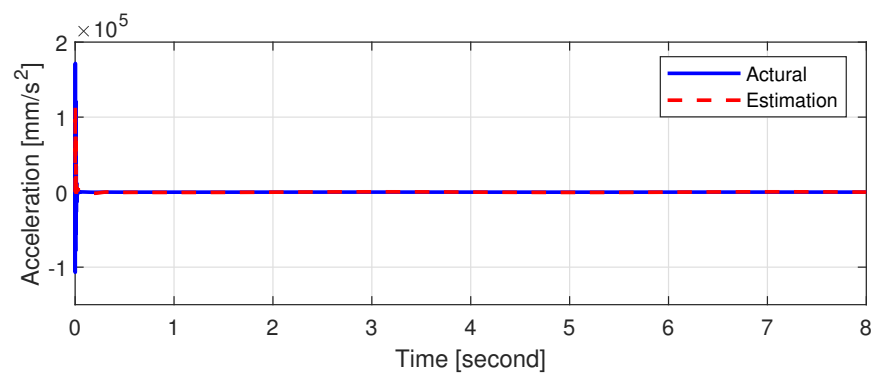

(c) Acceleration, $x_{3}$

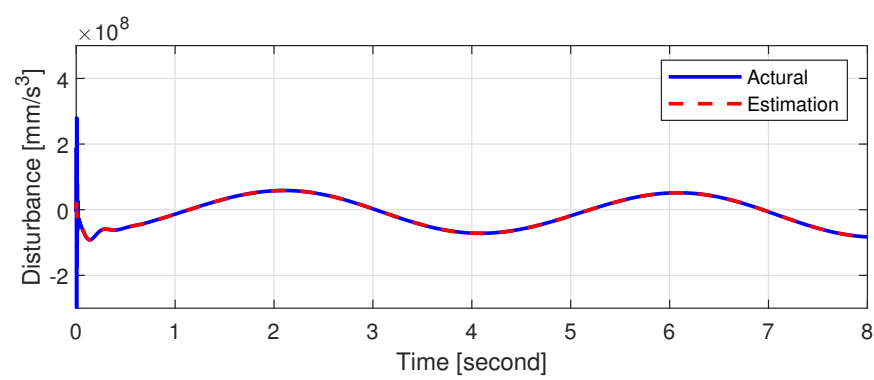

(d) Disturbance, $d$

Figure 4. Estimation performances of the proposed method. 


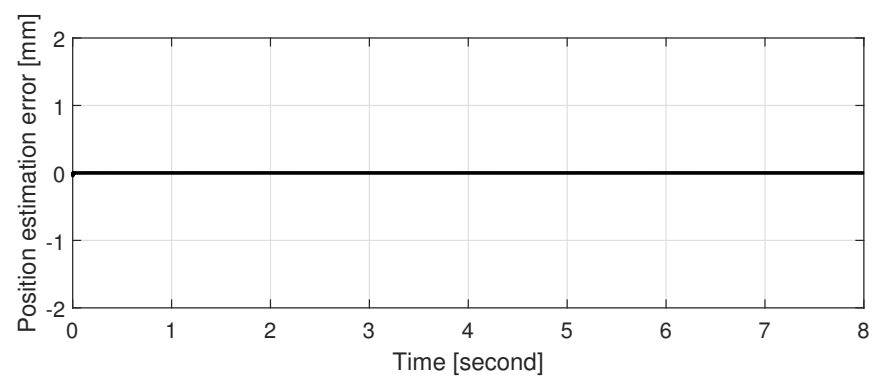

(a) Position estimation error

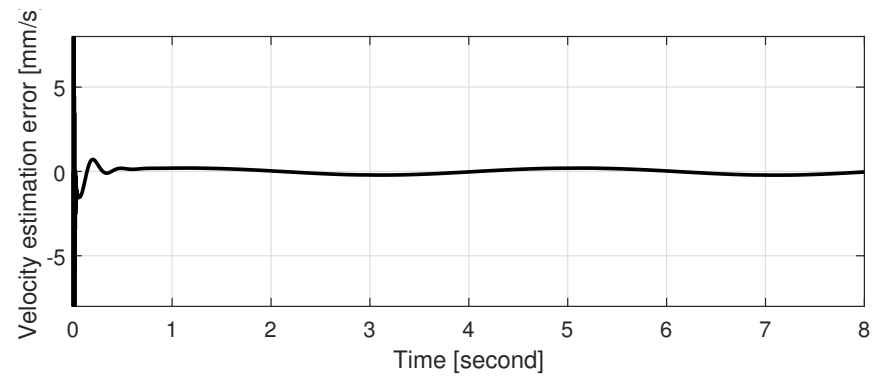

(b) Velocity estimation error

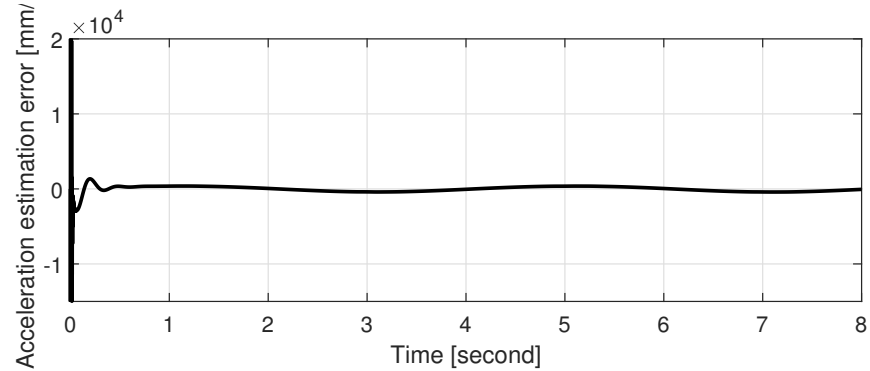

(c) Acceleration estimation error

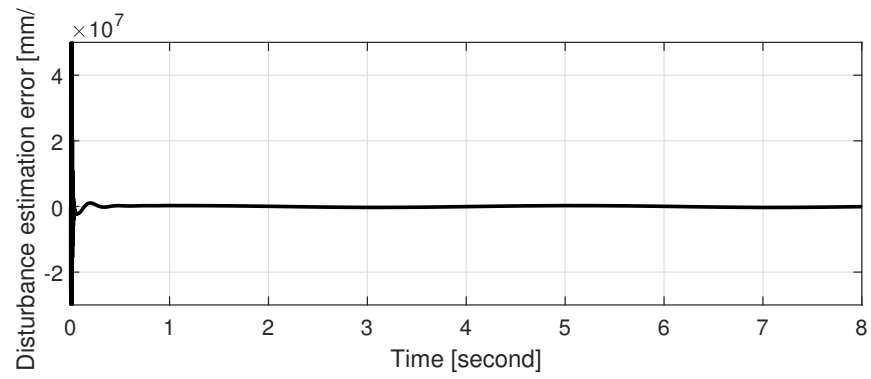

(d) Disturbance estimation error

Figure 5. Estimation errors of the proposed method.

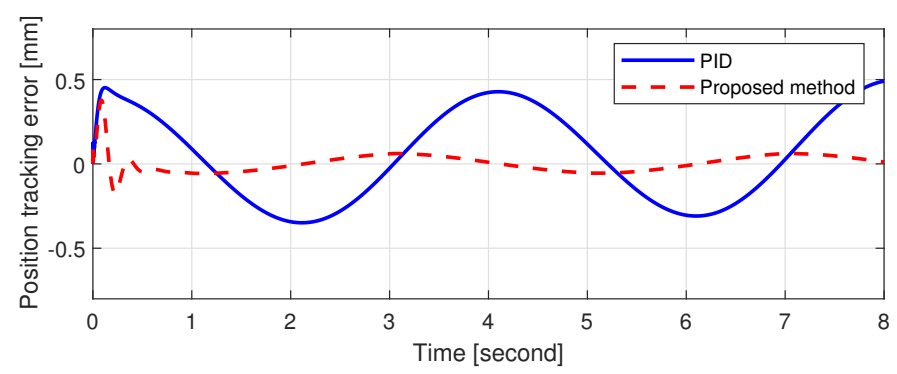

Figure 6. Position tracking error of the PID controller and the proposed method, $x_{1_{d}}-x_{1}$. 


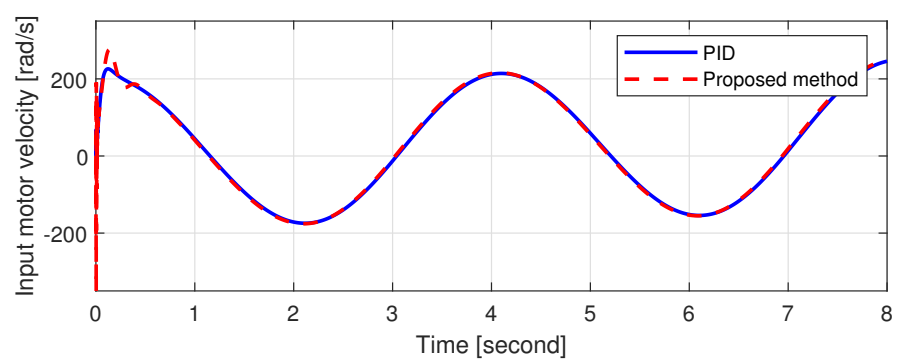

Figure 7. Control input of the PID controller and the proposed method, $\omega_{m}$.

\subsection{Step Desired Position Tracking}

The step desired position depicted in Figure 8 was used. The position tracking performances of the PID controller and the proposed method are shown in Figure 9. Due to step change in the desired position, large overshoots were observed in both methods. Thus, the large oscillation appeared in the PID controller. On the other hand, the position tracking error rapidly converged to zero due to the nonlinear damping effect of the proposed method.

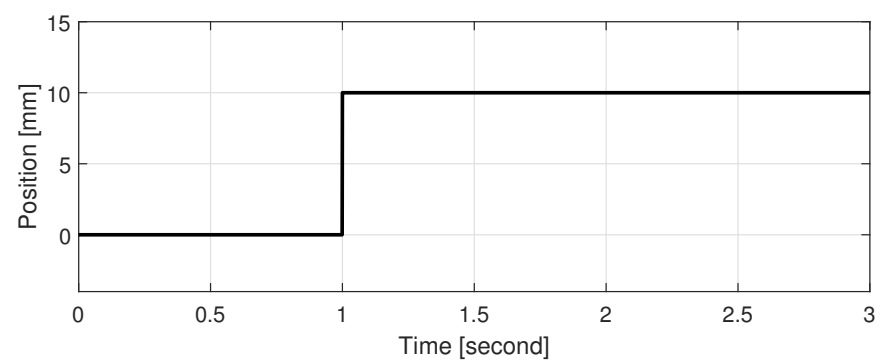

Figure 8. Desired position, $x_{1_{d}}$.

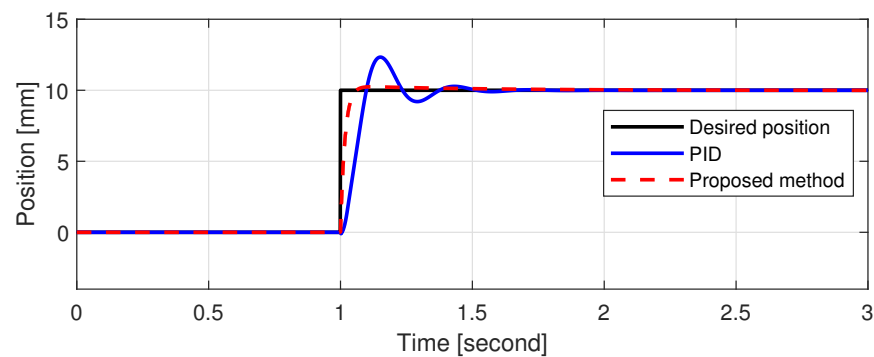

(a) Position tracking

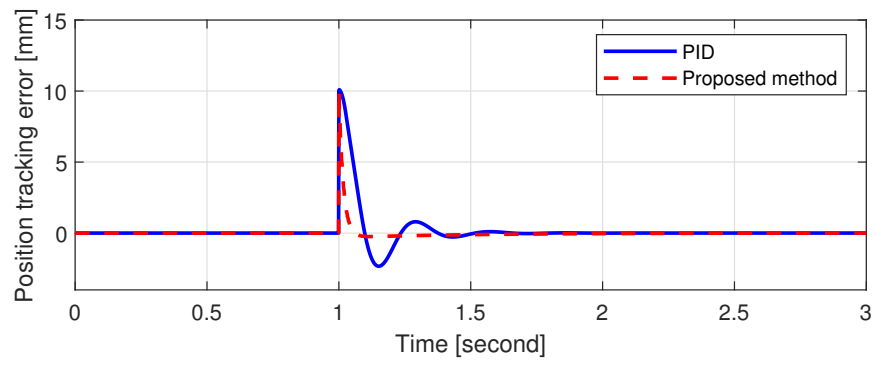

(b) Position tracking error

Figure 9. Position tracking performance of the PID controller and the proposed method.

\section{Conclusions}

Robust nonlinear position control with an ESO was developed for single-rod EHAs. A new EHA model was established for the controller design. The external disturbance, model, and input function uncertainties were lumped into a single disturbance. The ESO 
was developed to estimate the disturbance as well as the position, velocity, and acceleration. A robust nonlinear position controller was developed with the nonlinear damping gain via a backstepping procedure. The stability of the closed-loop system was mathematically proven using the ISS. The performance of the proposed method was validated using simulations. It was shown that the position tracking error was reduced by the proposed method using only position feedback. Furthermore, we see that the proposed method is robust against the model uncertainties and the external disturbance in the simulation results.

Author Contributions: Y.S.S. and W.K. designed the algorithm and developed the simulation; Y.S.S. and W.K. provided guidance in designing the algorithm; Y.S.S. and W.K. verified the simulation model and results. Both authors have read and agreed to the published version of the manuscript.

Funding: This research was supported partly by Basic Science Research Program through the National Research Foundation of Korea (NRF) funded by the Ministry of Education (2021R1A6A1A03043144) and partly by the Technology Innovation Program (20014121, Development of integrated Minimal Risk Maneuver Technology for Fallback system during autonomous Driving) funded By the Ministry of Trade, Industry \& Energy (MOTIE, Korea).

Institutional Review Board Statement: Not applicable.

Informed Consent Statement: Not applicable.

Data Availability Statement: Not applicable.

Conflicts of Interest: The authors declare that they have no conflict of interest.

\section{References}

1. Merrit, H.E. Hydraulic Control System; Wiley and Sons: New York, NY, USA, 1967.

2. Grabbel, J.; Ivantysynova, M. An investigation of swash plate control concepts for displacement controlled actuators. Int. J. Fluid Power 2005, 6, 19-36. [CrossRef]

3. Guan, C.; Pan, S. Adaptive sliding mode control of electro-hydraulic system with nonlinear unknown parameters. Control Eng. Prac. 2008, 16, 1275-1284. [CrossRef]

4. Kim, J.; Jin, M.; Choi, W.; Lee, J. Discrete time delay control for hydraulic excavator motion control with terminal sliding mode control. Mechatronics 2019, 60, 15-25. [CrossRef]

5. Tsao, T.-C.; Tomizuka, M. Robust adaptive and repetitive digital tracking control and application to a hydraulic servo for noncircular machining. J. Dyn. Syst. Meas. Control 1994, 116, 24-32. [CrossRef]

6. Hahn, H.; Piepenbrink, A.; Leimbach, K.-D. Input/output linearization control of an electro servo-hydraulic actuator. In Proceedings of the IEEE International Conference on Control Applications, San Francisco, CA, USA, 22-24 August 1994; pp. 995-1000.

7. Seo, J.; Venugopala, R.; Kenné, J.-P. Feedback linearization based control of a rotational hydraulic drive. Control Eng. Pract. 2007, 15, 1495-1507. [CrossRef]

8. Kim, W.; Shin, D.; Won, D.; Chung, C.C. Disturbance observer based position tracking controller in the presence of biased sinusoidal disturbance for electro-hydraulic actuators. IEEE Trans. Control Syst. Technol. 2013, 21, 2290-2298. [CrossRef]

9. Kim, W.; Won, D.; Tomizuka, M. Flatness-based nonlinear control for position tracking of electrohydraulic systems. IEEE/ASME Trans. Mechatron. 2015, 20, 197-206. [CrossRef]

10. Alleyne, A.; Liu, R. A simplified approach to force control for electro-hydraulic systems. Control Eng. Pract. 2000, 8, 1347-1356. [CrossRef]

11. Kaddissi, C.; Kenné, J.-P.; Saad, M. Identification and real-time control of an electrohydraulic servo system based on nonlinear backstepping. IEEE/ASME Trans. Mechatron. 2007, 12, 12-22. [CrossRef]

12. Kaddissi, C.; Kennè, J.-P.; Saad, M. Indirect adaptive control of an electrohydraulic servo system based on nonlinear backstepping. IEEE/ASME Trans. Mechatron. 2012, 16, 1171-1177. [CrossRef]

13. Song, B.; Lee, D.; Park, S.Y.; Baek, Y.S. Design and performance of nonlinear control for an electro-hydraulic actuator considering a wearable robot. Processes 2019, 7, 389. [CrossRef]

14. Ji, X.; Wang, C.; Zhang, Z.; Chen, S.; Guo, X. Nonlinear adaptive position control of hydraulic servo system based on sliding mode back-stepping design method. Proc. Inst. Mech. Eng. Part I J. Syst. Control Eng. 2021, 235, 474-485. [CrossRef]

15. Kim, W.; Won, D.; Shin, D.; Chung, C.C. Output feedback nonlinear control for electro-hydraulic systems. Mechatronics 2012, 22, 766-777. [CrossRef]

16. Kim, W.; Won, D. Observer based nonlinear position control using nonlinear coordinate transformation for single-rod electrohydrostatic actuator. Mathematics 2020, 8, 1273. [CrossRef]

17. Eryilmaz, B.; Wilson, B.H. Improved tracking control of hydraulic systems. J. Dyn. Syst. Meas. Control 2001, 123, 457-462. [CrossRef] 
18. Wang, L.; Book, W.J.; Huggins, J.D. Application of singular perturbation theory to hydraulic pump controlled systems. IEEE/ASME Trans. Mechatron. 2012, 17, 251-259. [CrossRef]

19. Alleyne, A.; Hedrick, J.K. Nonlinear adaptive control of active suspensions. IEEE Trans. Control Syst. Technol. 1995, 3, 94-101. [CrossRef]

20. Yao, B.; Bu, F.; Reedy, J.; Chiu, G.T.C. Adaptive robust motion control of single-rod hydraulic actuators: Theory and experiments. IEEE/ASME Trans. Mechatron. 2000, 5, 79-91.

21. Zeng, H.; Sepehri, N. Tracking control of hydraulic actuators using a LuGre friction model compensation. J. Dyn. Syst. Meas. Control 2008, 130, 014502. [CrossRef]

22. Kosut, R.L. Design of linear systems with saturating linear control and bounded states. IEEE Trans. Autom. Control 1983, 28, 121-124. [CrossRef]

23. Ang, K.H.; Chong, G.; Li, Y. PID control system analysis, design, and technology. IEEE Trans. Control Syst. Technol. 2005, 13, 559-576.

24. Armstrong-Helouvry, B.; Dupont, P.; de Wit, C. A survey of models, analysis tools and compensation methods for the control of machines with friction. Automutica 1994, 30, 1083-1138. [CrossRef] 